\title{
Anatomia do lenho de Croton urucurana Baill. (Euphorbiaceae) de solos com diferentes níveis de umidade
}

\author{
AGNES E. LUCHI ${ }^{1}$
}

(recebido: 29 de janeiro de 2003; aceito: 22 de janeiro de 2004)

\begin{abstract}
Wood anatomy of Croton urucurana Baill. (Euphorbiaceae) from soils in different moisture levels). Stem wood of Croton urucurana grown in flooded, moist, and dry soil areas, were studied. Twenty-two quantitative wood anatomical features of vessel elements, fibres, axial parenchyma, and rays were analysed. The characteristics average values for each soil type were statistically compared. Soil moisture data were obtained during one year for all the areas fortnightly; water soil retention curve was also obtained for characterisation of moisture levels. Fourteen anatomical features differed statistically at level of 5\% of significance, for specimens of two different places, at least regarding soil moisture. Ten features were significantly different for specimens in dry and flooded soil conditions. The frequency and the vessels diameter are larger in flooded specimens, and smaller in specimens from dry areas. The data reflects the well-known condition for specimens from dry soils; however they reveal a new fact: the frequency and vessels diameter increasing in the specimens that live in the stressing condition of flooded soils.
\end{abstract}

Key words - Croton urucurana, flooded soil, stem, wood anatomy

RESUMO - (Anatomia do lenho de Croton urucurana Baill. (Euphorbiaceae) de solos com diferentes níveis de umidade). Este estudo analisou a anatomia do lenho de indivíduos de Croton urucurana de áreas com o solo alagado, úmido e seco. A estrutura anatômica foi analisada quantitativamente através de medições de vinte e duas características, envolvendo os elementos de vasos, as fibras, os parênquimas axial e radial; o valor médio das características em cada tipo de solo foi comparado estatisticamente. Foram obtidos dados de umidade de solo, quinzenalmente, no período de um ano, de cada uma das três áreas, juntamente com a curva de retenção de água do solo para a caracterização do nível de umidade. Quatorze características, ao nível de 5\% de significância, apresentaram-se diferentes nos espécimes de pelo menos duas localidades diferentes de solo. De tais características, dez apresentaram-se significativamente diferentes entre os espécimes de solo da condição seca e alagada. A freqüência e o diâmetro dos vasos, apresentaram-se com médias maiores nos espécimes do solo alagado, e menores nos de solo seco. Tal dado revela um fato novo: o aumento da freqüência e do diâmetro dos vasos dos espécimes que vivem na condição estressante do solo alagado.

Palavras-chave - anatomia do lenho, caule, Croton urucurana, solo alagado

\section{Introdução}

As inter-relações entre a estrutura anatômica do lenho e parâmetros climáticos têm sido amplamente discutidas na literatura, porém com maior ênfase em espécies de vegetações mesófitas e xerófitas (Mattos Filho 1971 e 1989, Baas \& Carlquist 1985, BarajasMorales 1985, Gomes \& Muñiz 1986, Alves \& Angyalossy-Alfonso 2001). Pouco se conhece sobre os mecanismos que possibilitam a sobrevivência de espécies vegetais em áreas naturalmente inundáveis, que reduzem a disponibilidade de oxigênio para a planta, podendo afetar o crescimento de raízes e da sua parte aérea. As interpretações dos trabalhos que abordam os mecanismos de sobrevivência de plantas nessas áreas

1. Instituto de Botânica, Caixa Postal 4005, 01061-970 São Paulo, SP, Brasil. aeluchi@globo.com enfocam: as vias metabólicas, considerando que essas espécies ativam rotas que controlariam o mecanismo respiratório das raízes (Crawford 1978); a anatomia, que considera que as plantas apresentariam alterações morfoanatômicas possibilitando a difusão de oxigênio da parte aérea para as raízes (Armstrong 1979); ou ainda a ocorrência de uma interação de adaptações morfológicas, anatômicas e metabólicas (Joly 1991).

As principais modificações morfológicas e anatômicas que têm sido observadas em plantas alagadas são: redução do crescimento (Tang \& Kozlowski 1982, Newsome et al. 1982, Tsukahara \& Kozlowski 1985), hipertrofia da base do caule (Wample \& Reid 1979, Jackson \& Drew 1984, Yamamoto et al. 1995a, b, Pimenta et al. 1996), formação de raízes adventícias (Wenker et al. 1981, Hook 1984, Tsukahara \& Kozlowski 1985), hipertrofia de lenticelas (Newsome et al. 1982, Hook 1984, Topa \& McLeod 1986), formação de aerênquima na região cortical de caules e raízes (Kozlowski 1984), epinastia de folhas (Kozlowski 
1984, Sena-Gomes \& Kozlowski 1988) e senescência e abscisão de folhas (Tang \& Kozlowski 1982).

Entretanto, a totalidade dos trabalhos que enfocam as modificações causadas pelo alagamento é realizada em plântulas das espécies lenhosas e as análises das alterações anatômicas, geralmente, se restringem aos tecidos da casca. Trabalhos que analisam o lenho dos indivíduos adultos de espécies que ocorrem nessa área são inexistentes.

Croton urucurana Baill., conhecida popularmente por "sangue-da-água", "sangue-de-drago", "urucurana", entre outros, é espécie arbórea decídua, heliófila, pioneira, seletiva higrófita, característica de terrenos muito úmidos e brejosos, ocorrendo na Bahia, Rio de Janeiro, Minas Gerais e Mato Grosso do Sul até o Rio Grande do Sul (Lorenzi 1992). Assad-Ludewigs et al. (1989) verificaram que a espécie ocupa uma faixa marginal homogênea na praia do rio Moji-Guaçu, ocorrendo também além do limite externo da mata ciliar. Como planta pioneira adaptada à terrenos muito úmidos e brejosos, é indicada para plantios mistos em áreas ciliares degradadas.

Este estudo visou a análise anatômica do lenho de indivíduos adultos de Croton urucurana de solo alagado, úmido e seco, e a comparação estatística dos valores médios obtidos das características observadas.

\section{Material e métodos}

O estudo foi desenvolvido em três áreas (figura 1) situadas à margem do Rio Moji-Guaçu, na Estação Ecológica de Moji-Guaçu, Município de Moji-Guaçu, Estado de São Paulo. A área alagada (L1) localiza-se em terreno praticamente no nível do rio, na Mata do Português ( $47^{\circ} 08^{\prime}$ 'W e $22^{\circ} 17^{\prime} \mathrm{S}$ ), e por isso é constantemente inundada; as áreas úmida (L2) e seca (L3) localizam-se na Mata da Figueira ( $\left(7^{\circ} 11^{\prime}\right.$ W e $\left.22^{\circ} 16^{\prime} \mathrm{S}\right)$, sendo que L2 em terreno baixio, úmido mas não alagado e L3 em terreno elevado a 2,20 m em relação ao nível do rio, portanto mais seca que L2, que será, aqui referida apenas como seca.

Foram coletadas amostras de lenho, à altura do peito (1,30 $\mathrm{m}$ do solo), de três indivíduos de cada uma das áreas (tabela 1). Essas amostras estão depositadas na Xiloteca do Herbário do Estado "Maria Eneyda P. Kauffmann Fidalgo" (SPw) do Instituto de Botânica da Secretaria do Meio Ambiente do Estado de São Paulo.

De cada amostra de lenho foi retirado um corpo de prova da região mais interna da amostra, com cerca de $1,5 \mathrm{~cm}^{3}$. Eles foram amolecidos e secionados em micrótomo de deslize para obtenção das secções histológicas, que foram preparadas de acordo com as técnicas usuais, coradas com safranina ou verde rápido e montadas entre lâmina e lamínula com meio sintético (Johansen 1940, Sass 1951).

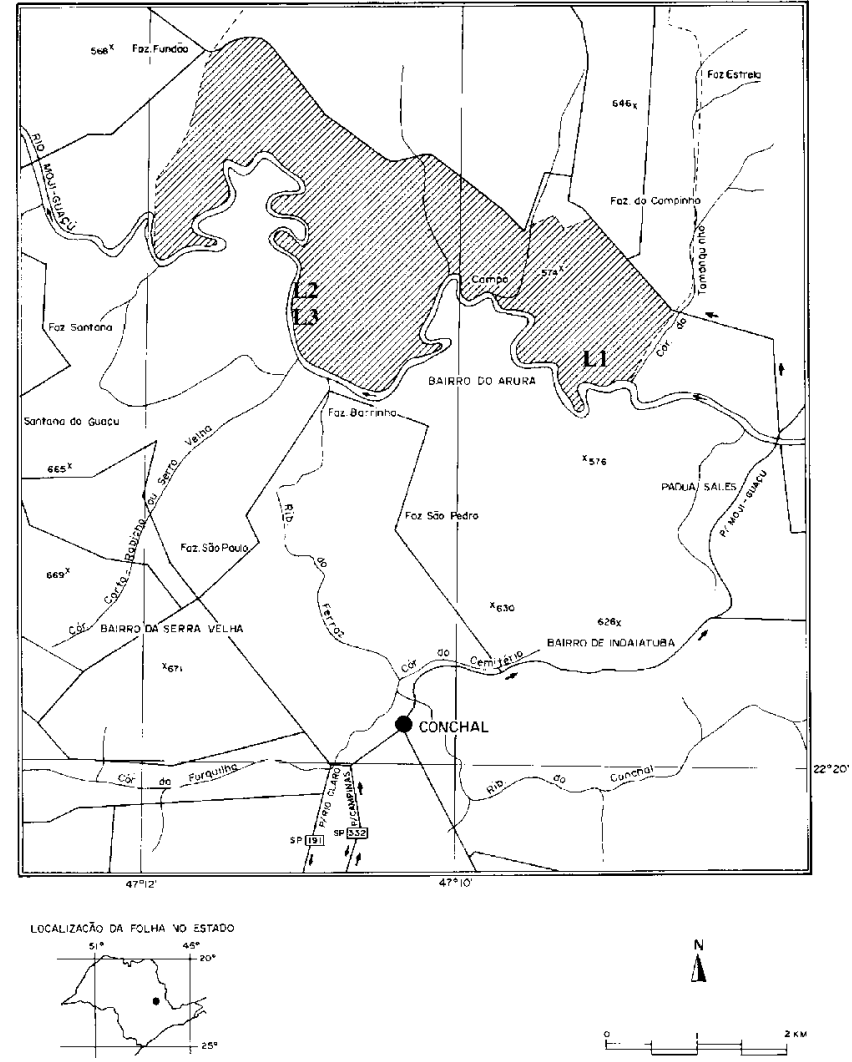

Figura 1. Localização das áreas de estudo, área alagada (L1), área úmida (L2) e área seca (L3), dentro da Estação Ecológica de Moji-Guaçu (área hachurada), às margens do Rio Moji-Guaçu - São Paulo, mapa confeccionado por Francisco José do Nascimento Kronka e equipe da Seção de Manejo e Inventário Florestal do Instituto Florestal (não publicado), modificado.

Figure 1. Localization of the study areas at Estação Ecológica de Moji-Guaçu (hatched), on the banks of the river MojiGuaçu: flooded area (L1), moist area (L2) and dry area (L3). Map by Francisco José do Nascimento Kronka and the team of "Seção de Manejo e Inventário Florestal" of "Instituto Florestal" (unpublished), modified.

A estrutura anatômica foi analisada quantitativamente através de 60 medições feitas em vinte e duas características envolvendo os vasos, as fibras, o parênquima axial e o radial: frequiência de vasos $(\mathrm{Fv})$, frequiência de vasos solitários $(\mathrm{Fs})$, frequiência de vasos geminados (Fg), diâmetro de vasos (Dv), comprimento de vasos $(\mathrm{Cv})$, diâmetro das pontoações intervasculares (Dpi), diâmetro das pontoações radiovasculares (Dpr), frequiência de raios (Fr), altura dos raios em milímetro (Arm), altura dos raios em número de células (Arc), largura dos raios nas fibras, em micrometro ( $\mathrm{LrFm})$, largura dos raios nas fibras, em número de células $(\mathrm{LrFc})$, largura dos raios no parênquima axial, em micrometro ( $\mathrm{LrPm}$ ), largura dos 
Tabela 1. Material analisado de Croton urucurana Baill.

Table 1. Analised specimens of Croton urucurana Baill.

\begin{tabular}{lccc}
\hline Área & SPw & Altura $(\mathrm{m})$ & Diâmetro $(\mathrm{cm})$ \\
\hline \multirow{3}{*}{ Seca } & 1473 & 7 & 11 \\
& 1474 & 7 & 10 \\
& 1475 & 8 & 16 \\
Úmida & 1459 & 6 & 7 \\
& 1460 & 5 & 7 \\
& 1472 & 5 & 6 \\
Alagada & 1385 & 10 & 20 \\
& 1458 & 9 & 8 \\
& 1461 & 9 & 11 \\
\hline
\end{tabular}

raios no parênquima axial, em número de células ( $\mathrm{LrPc})$, comprimento das fibras (Cf), diâmetro das fibras (Df), diâmetro do lume da fibra (Lf), espessura da parede da fibra (Epf), porcentagem de parênquima axial (Ppa), porcentagem de vasos $(\mathrm{Pv})$, porcentagem de fibras (Pf), porcentagem de raios (Pr). As porcentagens foram obtidas através da utilização de uma grade com 25 pontos, sobre a qual foi projetada a seção transversal da lâmina histológica e anotado o tipo de célula que caía sobre cada ponto, totalizando 60 áreas contíguas.

Esses dados foram analisados estatisticamente e submetidos à prova não paramétrica de Kruskal-Wallis, utilizando-se o nível de significância de $5 \%$ para os testes. Posteriormente utilizou-se a prova de Mann-Whitney para a comparação entre as médias.

Para o estudo da umidade do solo à base de peso $(\mu)$ (Reichardt 1985), aqui apresentado em porcentagem $(\mu \%)$, foram coletadas amostras a cada duas semanas, na profundidade de $0-10 \mathrm{~cm}$, em pontos padronizados em cada uma das áreas. As curvas de retenção de água, para 0,33 e 15 atm, foram obtidas através do pré secamento de amostras, utilizando Câmara de pressão de Richards.

\section{Resultados e Discussão}

A umidade do solo (figura 2) compreendida entre os dois níveis de retenção $(0,33$ e $15 \mathrm{~atm})$, representa a água disponível no solo a $10 \mathrm{~cm}$ de profundidade. Os valores acima da curva de 0,33 atm representam o excesso de água e os valores abaixo da curva de $15 \mathrm{~atm}$ o déficit hídrico. Nota-se que para a área seca, ocorreu um período de déficit hídrico, de cinco meses, acentuando-se no mês de setembro, observando-se também no mês de dezembro um pequeno déficit, apesar de estar em pleno período chuvoso; para a área úmida, os valores se apresentaram dentro do intervalo de água disponível, quando não apresentando um pequeno excedente hídrico; já para a área alagada os valores de umidade mantiveram-se, no período analisado, sempre com excedente hídrico.

A análise dos dados de umidade e da curva de retenção de água nas diferentes áreas revela as prováveis causas das características estruturais observadas nos espécimes de cada área estudada. $\mathrm{O}$ solo da área alagada, como já era de se esperar, não passou por nenhum período de déficit hídrico, assim como o da área úmida, que apesar de ter apresentado no mês de setembro nível de umidade abaixo da curva da capacidade de campo, permaneceu dentro do limite de disponibilidade hídrica. Nenhuma das duas áreas refletiu, no solo, o período anual de seca por que passa a região (Luchi 1998).

Os resultados da prova de Kruskal-Wallis, para as variáveis anatômicas, foram significantes para quatorze das vinte e uma características analisadas (tabela 2).

A figura 3 mostra as médias das características anatômicas que se apresentaram significantes em cada uma das áreas estudadas. Observa-se que dez características (Dv, Dpi, LrPm, Df, Lf, Epf, Pf, Cf, Fv e Fg) apresentaram-se significativamente diferentes entre os espécimes do solo da condição seca e alagada.

Das características anatômicas do lenho de Croton urucurana, que se apresentaram estatisticamente diferentes entre pelo menos duas das áreas, observa-se, nas figuras 4-6, a variação do diâmetro dos vasos (Dv), e da frequiência dos vasos ( $\mathrm{Fv})$, onde as maiores médias pertencem aos espécimes das áreas úmida e alagada.

A curva de retenção de água da área seca indica que o solo dessa área passa por um período anual de baixa disponibilidade hídrica, caracterizando a estação seca. As características anatômicas dos indivíduos dessa área, como a diminuição do diâmetro do elemento de vaso, coincidem com as condições já conhecidas de espécies de ambiente árido, que apresentam essa característica como uma adaptação à garantia do fluxo hídrico (Baas et al. 1983, Baas \& Carlquist 1985).

Entretanto, este estudo aponta dados novos para indivíduos arbóreos adultos: os espécimes do ambiente úmido e alagado (figura 3) apresentam elemento de vaso de grande diâmetro, associado a um aumento na freqüência; aqueles do ambiente seco (figuras 4-6) apresentam baixa freqüência de elementos de vaso. Yamamoto e Kozlowski (1987), trabalhando com indivíduos jovens de Thuya orientalis L., observaram traqueídes de maior diâmetro na porção submersa do caule. Tais dados divergem daqueles mencionados por Baas (1973), Barajas-Morales (1985) e Lindorf (1994), 
para quem as espécies de ambientes secos apresentam uma frequiência maior de vasos de menor diâmetro e as de ambiente úmido, frequiência menor de vasos.

A freqüência de vasos solitários foi estatisticamente similar tanto para os espécimes da área seca quanto para a área alagada, sendo que os da área úmida foram os que se distinguiram por apresentarem a maior concentração de vasos solitários. Já para vasos geminados, a maior frequiência foi observada nos indivíduos da área alagada (figura 3). Esse fato se opõe
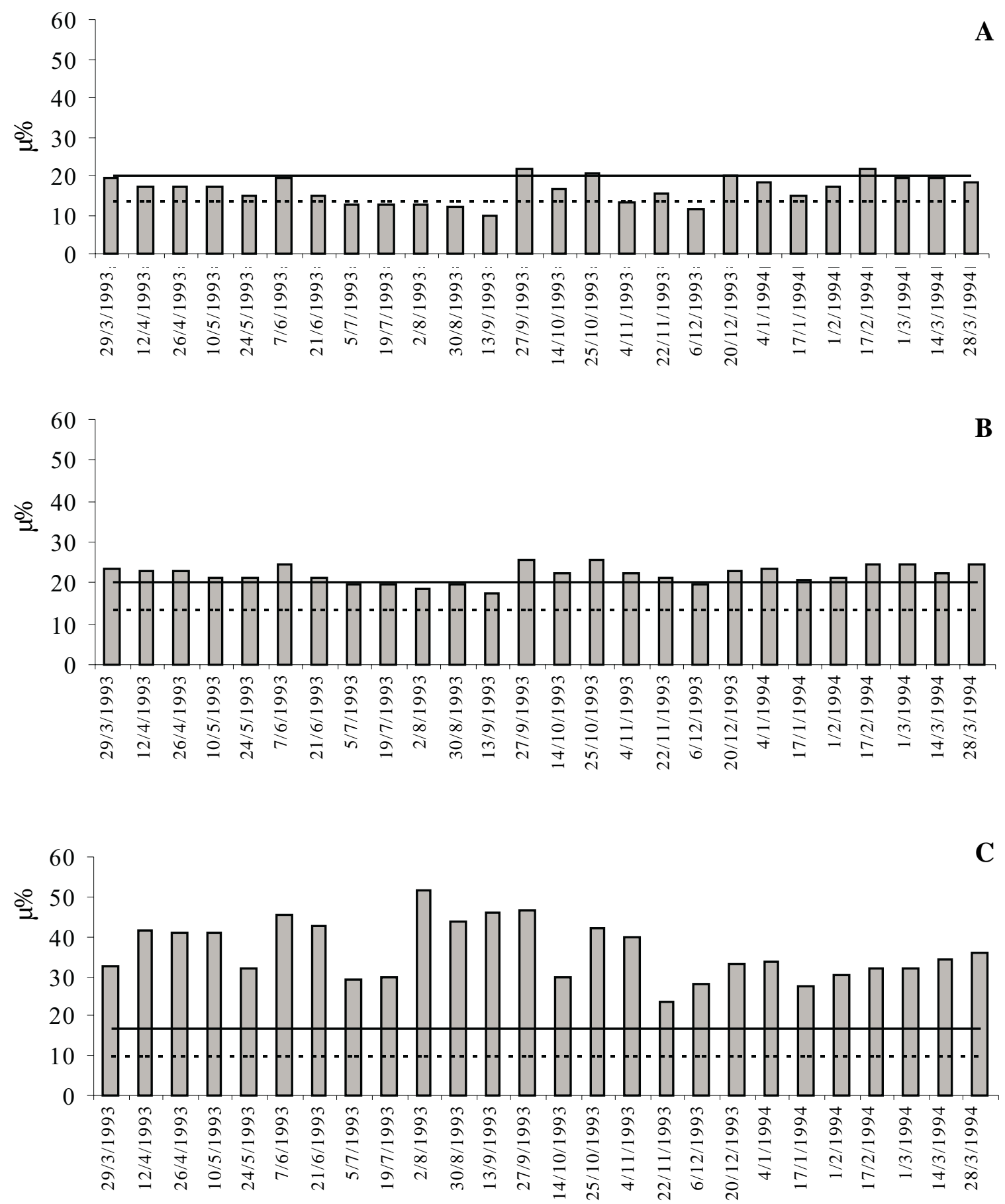

Figura 2. Média da umidade do solo ( $\mu \%$ ) das três áreas estudadas: seca (A), úmida (B) e alagada (C); curva de retenção da água a 15 atm (----) e $0,33 \mathrm{~atm}(-)$ para cada área.

Figure 2. Average of soil moisture ( $\mu \%)$ of the three study areas: dry (A), moist (B) and flooded (C); Water retention curves at $15 \mathrm{~atm}(----)$ and $0.33 \mathrm{~atm}(-)$ for each area. 
Tabela 2. Resultado da prova de Kruskal-Wallis ( $\mathrm{p}<0,05 \%)$.

Table 2. Results of Kruskal-Wallis proof ( $p<0,05 \%)$.

\begin{tabular}{lc}
\hline Variável & Probabilidade \\
\hline Freqüência de vasos & 0,0000 \\
Freqüência de vasos solitários & 0,0294 \\
Freqüência de vasos geminados & 0,0000 \\
Diâmetro de vasos & 0,0000 \\
Comprimento de elemento de vasos & 0,0000 \\
Diâmetro das pontoações intervasculares & 0,0011 \\
Largura dos raios no parênquima axial $(\mu \mathrm{m})$ & 0,0012 \\
Comprimento das fibras & 0,0000 \\
Diâmetro das fibras & 0,0000 \\
Diâmetro do lume das fibras & 0,0000 \\
Espessura da parede das fibras & 0,0000 \\
Porcentagem de vasos & 0,0220 \\
Porcentagem de fibras & 0,0160 \\
Porcentagem de raios & 0,0023 \\
\hline
\end{tabular}

ao observado por outros autores como Baas et al. (1983), Barajas-Morales (1985), Lindorf (1994) e Alves \& Angyalossy-Alfonso (2001) que encontraram vasos agrupados predominantemente em ambientes secos. Para vasos múltiplos de três a frequiência não foi representativa em nenhuma das áreas.

Com relação às fibras, as figuras 7-12 mostram as diferenças entre o diâmetro das fibras (Df) e o diâmetro do lume das fibras (Lf), onde as maiores médias ocorrem nos indivíduos das áreas úmida e alagada. Yamamoto et al. (1995a, b) obtiveram, em plântulas de Alnus japonica (Thunb.) Steud. e Fraxinus mandshurica Rupr., respectivamente, o aumento no diâmetro e no número de fibras formadas após o alagamento, atribuindo a ambos a estimulação do crescimento em diâmetro dos caules submersos. Porém nos espécimes adultos de C. urucurana, aqui analisados, observa-se uma média maior na porcentagem de fibras nos espécimes da área seca (figura 3), e menor na área alagada. Entretanto, a espessura da parede da fibra (Epf), que aqui apresentaram as menores médias tanto para a área úmida quanto para a alagada, também, é documentada por Yamamoto et al. (1995b) nas paredes das fibras formadas após o alagamento, em plântulas de Fraxinus mandshurica.

Com relação às demais características quantitativas, os resultados obtidos são também inéditos, apesar de haver poucas referências sobre a análise dessas características na literatura, que possibilitem uma discussão.
As figuras 13-15, exemplificam os diâmetros das pontoações intervasculares (Dpi) nos elementos de vaso dos espécimes das três áreas, onde as maiores médias pertencem aos espécimes da área alagada. Pelo fato de muitos pesquisadores considerarem essa característica como conservativa para espécie, gênero e mesmo família, ela não é abordada para análise com o ambiente.

As diferenças na largura, em micrômetros, dos raios no parênquima axial ( $\mathrm{LrPm})$, podem ser notadas nas figuras 16-18, onde os indivíduos das áreas úmida e alagada apresentam as menores médias. Outer \& Veenendaal (1976) também obtiveram os menores valores de largura de raios ( $\mathrm{LrPm})$ para a maioria das espécies de ambientes mésicos (floresta pluvial) e os maiores para aquelas de ambientes mais xéricos (savana).

Os espécimes da área alagada apresentam ainda as maiores médias para comprimento de fibras (Cf) (figura 3). As dimensões das células vegetais, dentre elas os elementos de vasos e as fibras, dependem de características genéticas e de fatores ecológicos, que podem interferir nas características ontogenéticas como o comprimento das iniciais fusiformes que originarão tanto os elementos de vaso quanto as fibras. Em condições de maior disponibilidade hídrica do ambiente, as iniciais fusiformes apresentarão maior turgor celular, resultando em elementos axiais de tamanho maior (Levitt 1980). A presença de fibras com comprimento maior nos espécimes da área alagada, corrobora essa tendência. Ficando, somente, em discussão os resultados obtidos para o comprimento de elemento de vaso $(\mathrm{Cv})$, onde as maiores médias ocorrem nos indivíduos das áreas seca e alagada, dados estes que, para a área seca, contrariam resultados obtidos por Carlquist \& Hoekman (1985), onde os elementos de vasos mais longos que a média foram observados em ecossistemas mésicos, e os de Baas et al. (1983) obtidos para espécies higrófilas.

Para a porcentagem de raios (Pr), também as maiores médias foram encontradas nos espécimes da área alagada (figura 9). A quantificação em porcentagem de tecidos foi feita por Luchi (1998) para espécies de duas matas ciliares, onde os resultados, para os raios, foram estatisticamente significantes nos espécimes de Eugenia repanda O. Berg e Inga vera Willd. para uma das matas ciliares, caracterizada, pela autora, como hidricamente desfavorável às espécies .

Além das diferenças encontradas nas características quantitativas, observam-se diferenças em algumas características qualitativas, como a presença de fibras com camada gelatinosa (figuras 7,10 ) e presença de delimitação de camadas de crescimento 


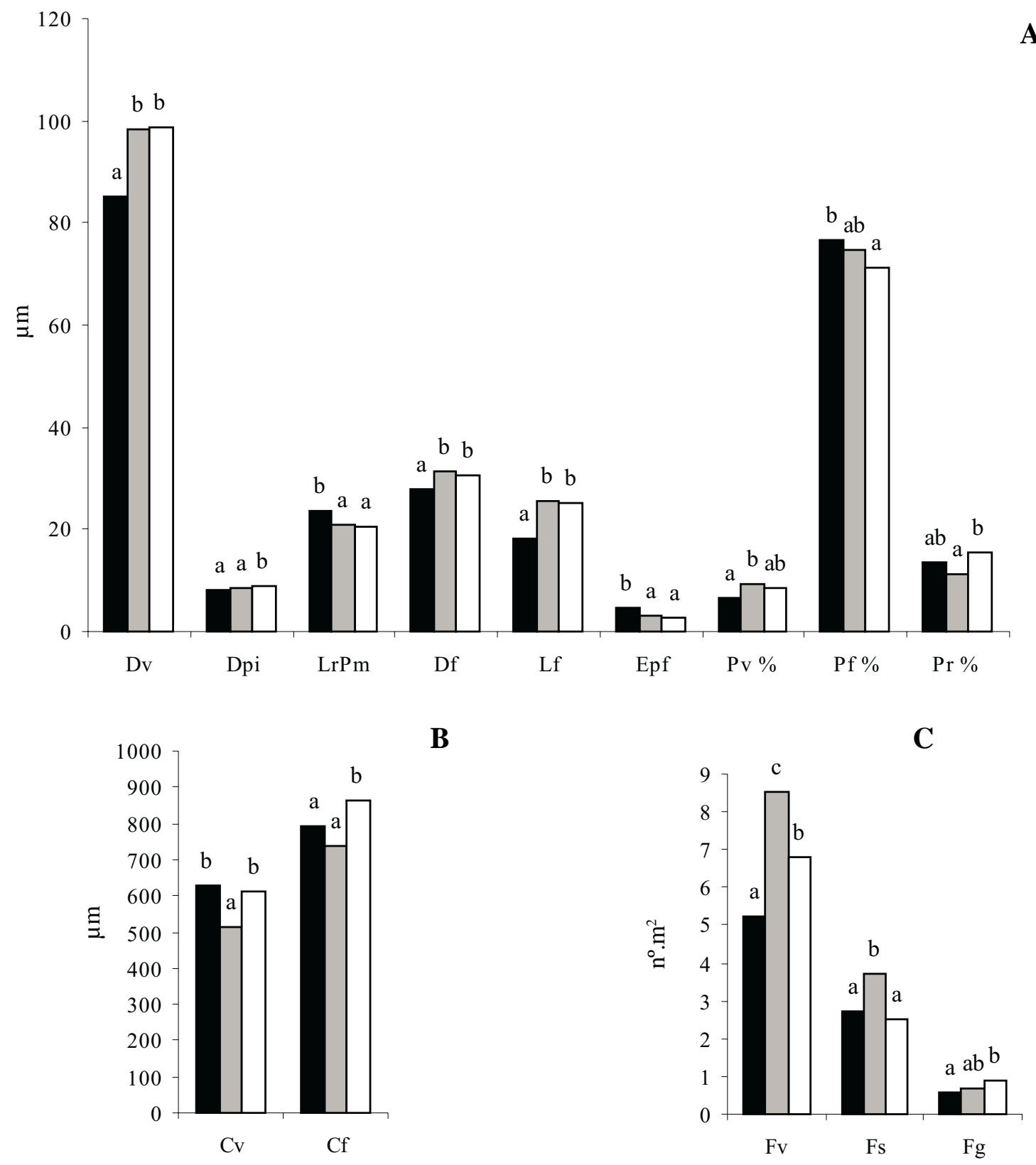

Figura 3. Resultado da prova de Mann-Whytney, onde as médias de cada característica anatômica são apresentadas para cada uma das áreas analisadas. Médias que diferem significativamente entre si, apresentam letras diferentes e aquelas que não diferem significantemente apresentam a mesma letra. $\mathbf{\square}=$ área seca; $\square=$ área úmida; $\square=$ área alagada; $D v=$ diâmetro de vasos; Dpi = diâmetro das pontoações intervasculares; $\mathrm{LrPm}=$ largura dos raios no parênquima axial $(\mu \mathrm{m}) ; \mathrm{Df}=$ diâmetro das fibras; $\mathrm{Lf}=$ diâmetro do lume das fibras das fibras; Epf = espessura da parede da fibra; $\mathrm{Pv}=$ porcentagem de vasos; $\mathrm{Pf}=$ porcentagem de fibras; $\mathrm{Pr}=$ porcentagem de raios; $\mathrm{Cv}=$ comprimento de vasos; $\mathrm{Cf}=$ comprimento das fibras; $\mathrm{Fv}=$ freqüência de vasos; $\mathrm{Fs}=$ frequiência de vasos solitários; $\mathrm{Fg}$ = frequiência de vasos geminados. As médias foram separadas em grupos A, B e C para melhor acomodar as escalas.

Figure 3. Results of Mann-Whytney proof for each anatomical feature for each analysed area. Averages followed by same letter are not significantly different. $\mathbf{\square}=$ dry area; $\square=$ moist area; $\square=$ flooded area; Dv = vessel diameter; Dpi= intervessel pits diameter; $\mathrm{LrPm}=$ ray width in axial parenchyma $(\mu \mathrm{m}) ; \mathrm{Df}=$ fibre diameter; $\mathrm{Lf}=$ diameter of fibre lumina; Epf $=$ fibre wall thickness; $\mathrm{Pv}=$ vessel percentage; $\mathrm{Pf}=$ fibre percentage; $\mathrm{Pr}=$ ray percentage $\mathrm{Cv}=$ vessel element length; $\mathrm{Cf}=$ fibre length; $\mathrm{Fv}=$ vessel per square millimeter; $\mathrm{Fs}=$ solitary vessel per square millimeter; $\mathrm{Fg}=2$-multiple vessel per square millimeter. The means were in different groups: A, B and $\mathrm{C}$ to adapt the scales. 

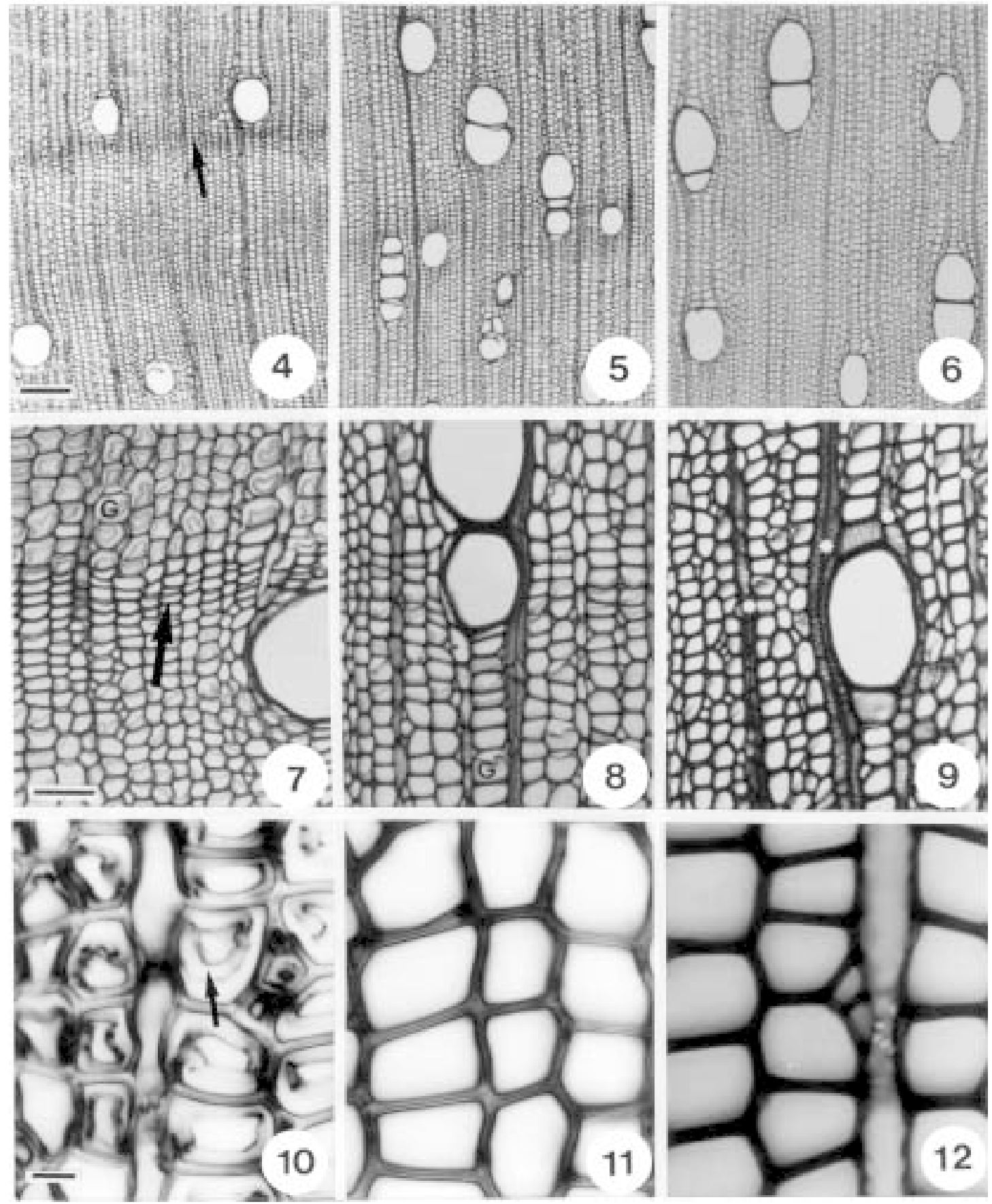

Figuras 4-12. Seções transversais de Croton urucurana. 4. Espécime da área seca, observar delimitação da camada de crescimento (seta). 5. Espécime da área úmida. 6. Espécime da área alagada. 7. Detalhe da figura 4, mostrando o achatamento das paredes radiais das fibras na delimitação da camada de crescimento (seta), e fibras gelatinosas (G). 8. Detalhe da figura 5, com algumas fibras gelatinosas $(\mathrm{G})$. 9. Detalhe da figura 6, observar a maior ocorrência de raios $(*)$. 10. Detalhe maior das fibras com a presença da camada gelatinosa (seta) destacada da parede secundária. 11. Detalhe da espessura de parede das fibras do espécime da área úmida. 12. Detalhe da espessura de parede das fibras do espécime da área alagada. Barras $=50 \mu \mathrm{m}(4-6), 50 \mu \mathrm{m}(7-9), 10 \mu \mathrm{m}(10-12)$.

Figures 4-12. Transversal sections of Croton urucurana. 4. Dry area specimen, note growth ring boundary (arrow). 5. Moist area specimen. 6. Flooded area specimen. 7. Detail of figure 4, showing flattened radial fibres wall in boundary growth ring (arrow) and gelatinous fibres (G). 8. Detail of figure 5, with gelatinous fibres (G). 9. Detail of figure 6, showing abundant rays $(*)$. 10. Lower magnification detail of fibres with gelatinous layer (arrow) detached from secondary wall. 11. Detail of fibre wall thickness from moist area specimen. 12. Detail of fibre wall thickness from flooded area specimen. Bars $=50 \mu \mathrm{m}(4-6), 50 \mu \mathrm{m}(7-9)$, $10 \mu \mathrm{m}(10-12)$. 
formada pelo achatamento das paredes radiais das fibras nos indivíduos da área seca (figura 4), não observadas nos espécimes das outras áreas.

A presença de um espessamento adicional de celulose às paredes secundárias das fibras confere maior espessura às mesmas e, por artefato de técnica essa camada adicional se desprende da parede lignificada, possibilitando uma melhor observação (figuras 7,10 ).
Devido a grande quantidade de celulose, hidrófila, as fibras de paredes gelatinosas são consideradas com função de reserva de água, uma vez que foram encontradas em sistemas subterrâneos de espécies de cerradão (Paviani 1978) e em maior quantidade no lenho de indivíduos de Copaifera langsdorffii Desf. do cerradão comparado com aqueles da floresta (Marcati et al. 2001). Sugere-se que a sua presença, nos
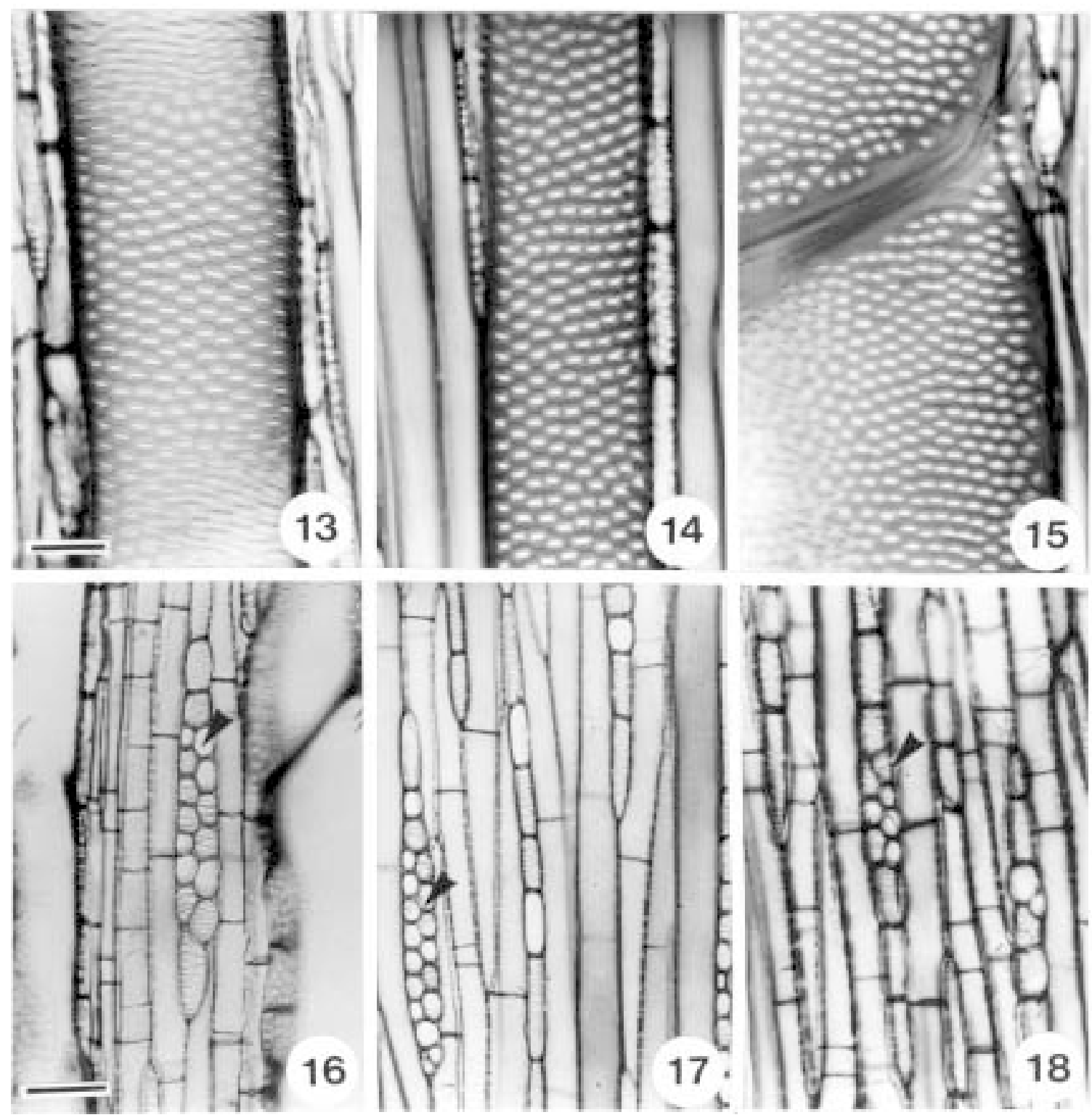

Figuras 13-18. Seções longitudinais tangenciais de Croton urucurana. 13-15. Pontoações intervasculares alternas de elemento de vaso. 13. Espécime área seca. 14. Espécime da área úmida. 15. Espécime da área alagada. 16-18. Largura dos raios (cabeça de seta). 16. Espécimes da área seca. 17. Espécime da área úmida. 18. Espécime da área alagada. Barras = $30 \mu \mathrm{m}(13-15), 50 \mu \mathrm{m}$ (16-18).

Figures 13-18. Longitudinal tangential sections of Croton urucurana. 13-15. Alternate intervessel pits of vessel element. 13. Dry area specimen. 14. Moist area specimen. 15. Flooded area specimen. 16-18. Ray width (arrowhead). 16. Dry area specimen. 17. Moist area specimen. 18. Flooded area specimen. Bars $=30 \mu \mathrm{m}(13-15), 50 \mu \mathrm{m}(16-18)$. 
indivíduos de C. urucurana da área seca, também esteja relacionada ao armazenamento de água, como um mecanismo de resistência ao período de baixa disponibilidade hídrica, caracterizado na curva de retenção de água dessa área. A presença de delimitação de camadas de crescimento, observada apenas nos espécimes de área seca, também reflete o período de seca anual pelo qual passa a região.

Os resultados de umidade de solo, juntamente com as curvas de retenção de água deixam claro o estado de disponibilidade hídrica de cada área analisada, e os dados estatísticos da estrutura anatômica dos espécimes adultos de $C$. urucurana, que se mostraram significantemente diferentes, principalmente entre os indivíduos das áreas alagada e seca, indicam que a espécie apresenta estratégias anatômicas distintas de tolerância aos estresses ambientais, estratégias que deverão ser analisadas para outras espécies para uma discussão mais ampla.

\section{Referências bibliográficas}

ALVES, E.S. \& ANGYALOSSY-ALFONSO, V. 2001. Ecological trends in the wood anatomy of some Brazilian species. 1. Growth rings and vessels. IAWA Journal 21:31-40.

ARMSTRONG, W. 1979. Aeration in higher plants. Advances in Botanical Research 7:226-332

ASSAD-LUDEWIGS, I.Y., PINTO, M.M., SILVA FILHO, N.L., GOMES, E.C. \& KANASHIRO, S. 1989. Propagação, crescimento e aspectos ecofisiológicos em Croton urucurana Baill. (Euphorbiaceae), arbórea nativa pioneira de mata ciliar. In Simpósio de Matas Ciliares (L.M. Barbosa, ed.). Fundação Cargill, Campinas, p.285-298.

BAAS, P. 1973. The wood anatomical range in Ilex (Aquifoliaceae) and its ecological and phylogenetic significance. Blumea 21:193-258.

BAAS, P. \& CARLQUIST, S. 1985. A comparison of the ecological wood anatomy of the floras of Southern California and Israel. IAWA Bulletin, n.s. 6:349-353.

BAAS, P., WERKER, E.E. \& FAHN, A. 1983. Some ecological trends in vessel characters. IAWA Bulletin, n.s. 4:141-159.

BARAJAS-MORALES, J. 1985. Wood structural differences between trees of two tropical forests. IAWA Bulletin, n.s. 6:355-364.

CRAWFORD, R.M.N. 1978. Metabolic adaptations to anoxia. In Plant life in anaerobic environments (R.M.N. Crawford \& D.D. Hook, eds.). Ann Arbor Science, Michigan, p.119-136.

GOMES, A.V. \& MUÑIZ, G.I.B. 1986. Wood structure and ultrastructure of Prosopis caldenia, P. chilensis and $P$. juliflora and influence of ecological factors. II International Conference on Prosopis. FAO, Recife, p.195-216.
HOOK, D.D. 1984. Adaptation to flooding with fresh water. In Flooding and plant growth (T.T. Kozlowski, ed.). Academic Press, London, p.265-294

JACKSON, M.B. \& DREW, M.C. 1984. Effects of flooding on growth and metabolism of herbaceous plants. In Flooding and plant growth (T.T. Kozlowski, ed.). Academic Press, London, p.47-128.

JOHANSEN, D.A. 1940. Plant microtechnique. McGraw-Hill, New York.

JOLY, C.A. 1991. Flooding tolerance in tropical trees. In Plant life under oxigen deprivation (M.B. Jackson, D.D. Davies \& H. Lambers, eds.). Academic Publishing, The Hague, p.23-34.

KOZLOWSKI, T.T. 1984. Responses of woody plants to flooding. In Flooding and plant growth (T.T. Kozlowski, ed.). Academic Press, London, p.129-163.

LEVITT, J. 1980. Responses of plants to environmental stresses. v.1 - Chiling, freezing and high temperature stresses. Physiological Ecology - a series of monographs, texts, and treatises. Academic Press, New York.

LINDORF, H. 1994. Eco-anatomical wood features of species from a very dry tropical forest. IAWA Journal 15:361-376.

LORENZI, H. 1992. Árvores brasileiras: manual de identificação e cultivo de plantas arbóreas nativas do Brasil. Plantarum, Nova Odessa.

LUCHI, A.E. 1998. Periodicidade de crescimento em Hymenaea courbaril L. e anatomia ecológica do lenho de espécies de mata ciliar. Tese de doutorado, Universidade de São Paulo, São Paulo.

MARCATI, C.R., ANGYALOSSY-ALFONSO, V. \& BENETATI, L. 2001. Anatomia comparada do lenho de Copaifera langsdorffii Desf. (Leguminosae-Caesalpinoideae) de floresta e cerradão. Revista Brasileira de Botânica 24:311-320.

MATTOS FILHO, A. 1971. Estudo comparativo entre duas espécies de Leguminosae latescentes do cerrado e da caatinga. Rodriguesia 38:9-33.

MATTOS FILHO, A. 1989. Estudo morfológico da madeira do par de espécies vicariantes de Pithecellobium anajulidae Riz. e Pithecellobium tortum Mart. (Leguminosae - Mimosoideae). Revista Brasileira de Biologia 48:143-154.

NEWSOME, R.D., KOZLOWSKI, T.T. \& TANG, Z.C. 1982. Responses of Ulmus americana seedling to flooding of soil. Canadian Journal of Botany 60:1688-1695.

OUTER, R.W. \& VEENENDAAL, W.L.H. 1976. Variation in wood anatomy of species with a distribution covering both rain forest and savanna areas of the Ivory Coast, West-Africa. In: Wood structure in biological and technological research (P. Baas, A.J. Bolton \& D.M. Catling, eds.). Leiden Botanical Series. n. 3. Leiden University Press, Leiden, p.182-195.

PAVIANI, T.I. 1978. Anatomia vegetal e cerrado. Ciência e Cultura 30:1076-1086. 
PIMENTA, J.A., MEDRI, M.E., BIANCHINI, E., MULLER, C., OKAMOTO, J.M., FRANSCISCONI, L.M.J. \& CORREA, G.T. 1996. Aspectos da morfoanatomia e fisiologia de Jacaranda puberula Cham. (Bignoniaceae) em condições de hipoxia. Revista Brasileira de Botânica 19:215-220.

REICHARDT, K. 1985. Processo de transferência no Sistema Solo - Planta - Atmosfera. $4^{a}$ ed., Fundação Cargill, Campinas.

SASS, J.E. 1951. Botanical microtechnique. The Iowa State College Press, Ames.

SENA-GOMES, A.R. \& KOZLOWSKI, T.T. 1988. Physiological and growth responses to flooding of seedling of Hevea brasiliensis. Biotropica 20:286-293.

TANG, Z.C. \& KOZLOWSKI, T.T. 1982. Some physiological and growth responses of Betula papyrifera seedling to flooding. Physiologia Plantarum 55:415-420.

TOPA, M.A. \& MCLEOD, K.W. 1986. Aerenchyma and lenticel formation in pine seedlings: A possible avoidance mechanism to anaerobic growth conditions. Physiologia Plantarum 68:540-550.
TSUKAHARA, H. \& KOZLOWISKI, T.T. 1985. Importance of adventitious roots to growth of flooded Platanus occidentalis seedlings. Plant \& Soil 88:123-132.

WAMPLE, R.L. \& REID, D.M. 1979. The role of endogenous auxins and ethylene in formation of adventitious roots and hypocotyl hypertrophy in flooded sunflower plants (Helianthus annuиs). Physiologia Plantarum 45:219-226.

WENKER, W., FAUSEY, N.R. \& WATTERS, H.D. 1981. Flooding responses in Zea mays L. Plant and Soil 62:351-366.

YAMAMOTO, F. \& KOSLOWSKI, T.T. 1987. Effect of flooding of soil on growth, stem anatomy, and ethylene production of Thuya orientalis seedlings. IAWA Bulletin, n. s. 8:21-29.

YAMAMOTO, F., SAKATA, T. \& TEREZAWA, K. 1995a. Growth, morphology, stem anatomy, and ethylene production in flooded Alnus japonica seedlings. IAWA Journal 16:47-59.

YAMAMOTO, F., SAKATA, T. \& TEREZAWA, K. 1995b. Physiological, morphological and anatomical responses of Fraxinus mandshurica seedlings to flooding. Tree Physiology 15:713-719. 